\title{
Seizure Prophylaxis in Traumatic Brain Injury: A Comparative Study of Levetiracetam and Phenytoin Cerebrospinal Fluid Levels in Trauma Patients with Signs of Increased Intracranial Pressure Requiring Ventriculostomy
}

Bailey Zampella ${ }^{1}$, Tye Patchana ${ }^{1}$, James G. Wiginton IV ${ }^{1}$, James Brazdzionis ${ }^{1}$, Marc Billings ${ }^{1}$, Benjamin Archambeau $^{2}$, Alfonso Avila ${ }^{2}$, Jeffrey Wang ${ }^{3}$, Margaret Wacker ${ }^{4}$, Dan E. Miulli ${ }^{1}$

1. Neurosurgery, Riverside University Health System Medical Center, Moreno Valley, USA 2. Emergency Medicine, Arrowhead Regional Medical Center, Colton, USA 3. Pharmaceutical Sciences, Western University of Health Sciences, Pomona, USA 4. Neurosurgery, Arrowhead Regional Medical Center, Colton, USA

Corresponding author: Tye Patchana, tyepatchana@gmail.com

\section{Abstract}

\section{Background}

One of the most common life-threatening injuries to trauma patients arriving in the emergency department (ED) is traumatic brain injury (TBI). Traditionally, intravenous medications have been given as seizure prophylaxis in patients demonstrating signs of increased intracranial pressure (ICP), as post-traumatic seizures in trauma patients are associated with higher morbidity and mortality. Medications traditionally given for this indication such as phenytoin have been established to reach therapeutic levels in the cerebrospinal fluid (CSF) quickly and are effective in preventing post-traumatic seizures but often have a large side-effect profile. A newer medication that is being used for seizure prophylaxis in patients with epilepsy is levetiracetam. Levetiracetam typically has a better side effect profile, but it has not been demonstrated that the drug reaches therapeutic levels in the CSF as quickly as phenytoin. Studies have shown levetiracetam and phenytoin to be equivocal in the prevention of post-TBI seizure prophylaxis.

\section{Methods}

This was a prospective, randomized, case-control study at a Level II trauma center of adult patients (age >/= 18 years) who suffered severe TBI (sTBI) requiring the placement of an external ventricular drain (EVD) from May 2017 to June 2018. Twelve patients were randomly placed into one of two groups for the administration of antiepileptic medication (either levetiracetam or phenytoin), allowing for the subsequent serial collection of CSF for the analysis of therapeutic levels of antiepileptic medications. Levetiracetam or phenytoin was administered at standardized fixed doses per our neurosurgical center standard protocol. CSF was collected before either drug was administered, 60 minutes after completion of administration and 360 minutes after completion of drug administration. Data analysis was performed to compare the time frame for which

Received 07/31/2019

Review began 09/06/2019 Review ended 09/11/2019 Published 09/27/2019

\section{(c) Copyright 2019}

Zampella et al. This is an open access article distributed under the terms of the Creative Commons Attribution License CC-BY 3.0., which permits unrestricted use, distribution, and reproduction in any medium, provided the original author and source are credited. therapeutic levels of the medications were achieved in the CSF. The published steady-state and therapeutic CSF level of levetiracetam is $32 \mathrm{mcg} / \mathrm{ml}$ and phenytoin is $2 \mathrm{mcg} / \mathrm{ml}$.

\section{Results}

A trend was observed in which the closer the fixed dosage approximated the weight-based dosing of phenytoin, the more their CSF phenytoin level increased (and approximated the therapeutic range) with an associated R-squared value of 0.6274 . This trend was not found in patients receiving levetiracetam.

\section{Conclusions}

Levetiracetam does not reach levels needed for seizure prophylaxis in human CSF when loaded at standard dosing regimens in the acute setting. Phenytoin does reach levels needed for seizure prophylaxis in human CSF with standardized regimen dosing when dosages approximate weight-based dosing. If needed, in the acute setting phenytoin should have additional doses given prior to six hours after the loading dose to achieve therapeutic CSF levels.

Categories: Neurology, Neurosurgery, Trauma

Keywords: pharmacodynamics, keppra, levetiracetam, dilantin, phenytoin, traumatic brain injury, seizure, seizure prophylaxis, cerebrospinal fluid

\section{Introduction}

Traumatic brain injury (TBI) remains a major health problem in the United States, with more than one 
million injuries occurring per year, causing not only death but also long-term disability [1-2]. For patients who sustain a severe TBI (sTBI), as defined by a Glasgow Coma Score (GCS) of eight or less, antiepileptic therapy (AED) significantly decreases the incidence of early post-traumatic seizures [2-3]. Per our center's standard protocol, based on Brain Trauma Foundation guidelines, an external ventricular drain (EVD) is placed in patients who suffer sTBI, as defined by a GCS of eight or less [4]. In addition to monitoring and treating elevated intracranial pressures, the placement of an EVD allows for routine and regular monitoring of CSF constituents as surveillance. The administration of intravenous antiepileptic medications, such as levetiracetam or phenytoin, in the emergency department and/or intensive care unit (ICU), serve as prophylaxis in patients with sTBI who may develop increased intracranial pressure (ICP). Levetiracetam, a newer agent than phenytoin, has shown similar outcomes to decrease post-traumatic seizures [5]. Although medications traditionally given for this indication, such as phenytoin, have been established to reach therapeutic levels in cerebrospinal fluid (CSF) quickly and are effective in preventing early post-traumatic seizures, they often have a large side-effect profile [6]. Despite this, the prevention of post-traumatic seizures is critical, as post-traumatic seizures in sTBI trauma patients are associated with higher morbidity and mortality [6].

Per the landmark paper by Temkin, the use of phenytoin in sTBI shows a reduction in early post-traumatic seizures when compared to placebo [3]. An alternative medication that is being used for seizure prophylaxis in patients with sTBI is levetiracetam. Levetiracetam is not metabolized via the hepatic cytochrome p450 system, has a half-life of approximately six to eight hours, and two-thirds is excreted unaltered in the urine [5-6]. Levetiracetam has been found to have a lower side-effect profile, but currently, there are not significant evidentiary studies to demonstrate how long it takes the drug to reach therapeutic levels in the human CSF [7]. Currently, research is being conducted to investigate the dosing of levetiracetam (standard versus low dose, as well as in comparison to phenytoin) in regard to seizure prophylaxis after sTBI; however, further studies are needed [8]. The recent use of levetiracetam as seizure prophylaxis has increased due to the ease of dosing and a lack of need for drug level monitoring $[5,9]$. Multiple studies currently show similar outcomes based on the use of levetiracetam versus phenytoin for decreasing long-term post-traumatic seizure $[5,9-11]$. However, there is no current study that addresses the acute therapeutic level of levetiracetam in the CSF for effective seizure prophylaxis. The objective of our study is to acutely evaluate the CSF levels of levetiracetam versus phenytoin in patients with sTBI.

\section{Materials And Methods}

This was a prospective, non-blinded, randomized study conducted as a collaborative effort by the neurosurgery department and the emergency department at a level II trauma center from May 2017 to June 2018.

A total of 12 patients (six females, six males) were enrolled. Inclusion criteria included either male or female patients 18 years or older who presented with sTBI and required the placement of an EVD. Exclusion criteria included any patients under the age of 18 years old, patients with a known seizure disorder and who take antiepileptic medication regularly, patients with a known allergy to either levetiracetam or phenytoin, or those who were deemed to have a direct contraindication to either medication as established by the drug manufacturer. Patients were identified as having sTBI by a GCS of eight or less, requiring the placement of an EVD according to the guidelines for the management of severe head injury, and were randomly assigned to one of the two groups of the study. The levetiracetam group (six patients) were given only intravenous levetiracetam (standard dosing at our institution $1000 \mathrm{mg}$ loading dose, followed by $1000 \mathrm{mg}$ BID) and the phenytoin group (six patients) were given only intravenous phenytoin (standard dosing at our institution $1000 \mathrm{mg}$ loading dose, followed by $200 \mathrm{mg}$ BID typically scheduled at 0900/2100 first dose given at the next scheduled time).

Serial CSF samples were drawn at designated times, including prior to the administration of antiepileptic medication, which served as a baseline control, and then subsequently at one hour and six hours after the administration of antiepileptic medication. Samples were labeled with predetermined randomized numbers and stored in a -20 degree Celsius refrigerator and then was evaluated with high-performance liquid chromatography (HPLC).

The HPLC method for the simultaneous quantification of levetiracetam and phenytoin was developed using a Hewlett-Packard 1050 HPLC system (California, US), consisting of a degasser, a quaternary pump, and an autosampler. The system also included a diode array detector and a computer running Chemstation software (Rev. A.08.03; Agilent Technologies, California, US) for data acquisition and processing. Chromatographic separation was performed using a Waters Symmetry ${ }^{\circledR}$ C18 column $(4.6$ x $150 \mathrm{~mm}, 5 \mu \mathrm{m}$; Waters Corporation, Massachusetts, US). The analytes were quantified by ultraviolet (UV) detection at $220 \mathrm{~nm}$. The mobile phase comprised water (A) and acetonitrile (B) and the flow rate was $1.0 \mathrm{ml} / \mathrm{min}$. The elution began with $2 \% \mathrm{~B}$ followed by programmed elution: linear gradient to $25 \% \mathrm{~B}$ from zero to six min; linear gradient to $95 \% \mathrm{~B}$ from six to $12 \mathrm{~min}$; linear gradient to $2 \% \mathrm{~B}$ from 12 to $13 \mathrm{~min}$; and isocratic at $2 \% \mathrm{~B}$ from 13 to $15 \mathrm{~min}$. The total run time was $15 \mathrm{~min}$. Under these conditions, levetiracetam and phenytoin eluted from the column at 5.83 and $9.66 \mathrm{~min}$, respectively. Samples were prepared using a protein precipitation procedure. Briefly, to a volume of $100 \mu \mathrm{l} \mathrm{CSF}$ sample in a microcentrifuge tube $(1.7 \mathrm{ml})$ was added $10 \mu \mathrm{l} 50 \%$ methanol, followed by $490 \mu \mathrm{l}$ acetonitrile. The mixture was separated by centrifugation $(140,000 \mathrm{rpm})$ at room temperature for 15 


\section{Cureus}

min. The supernatant $(580 \mu \mathrm{l})$ was transferred to a borosilicate glass tube $(12 \times 75 \mathrm{~mm})$ and evaporated to dryness with a stream of dry air. The residue was redissolved in 50\% methanol (100 $\mu \mathrm{l})$ and loaded into a plastic vial insert and $20 \mu \mathrm{l}$ was injected into the HPLC system. Calibration curves were constructed from 0.5 to $20 \mu \mathrm{g} / \mathrm{ml}$ for both levetiracetam and phenytoin with good linearity $(\mathrm{r}>0.99)$. Data analysis was completed using Microsoft Excel ${ }^{\circledR}$ (Microsoft Corporation, Washinton, US).

\section{Results}

Twelve patients were included for analysis with HPLC. Of the 12, six patients received levetiracetam and six received phenytoin. HPLC results were tabulated to obtain CSF concentrations as listed in Tables 1-2. Goal concentrations were selected for $2 \mu \mathrm{g} / \mathrm{mL}$ for phenytoin and $12 \mu \mathrm{g} / \mathrm{mL}$ for levetiracetam [12-13]. Goal phenytoin and levetiracetam doses were calculated according to established norms of dosing for phenytoin and levetiracetam, respectively $17 \mathrm{mg} / \mathrm{kg}$ and $20 \mathrm{mg} / \mathrm{kg}$ [14]. Differences between goal CSF phenytoin and levetiracetam concentrations were calculated by subtracting the goal concentrations of CSF phenytoin and levetiracetam from the measured CSF phenytoin and levetiracetam measured by HPLC.

\begin{tabular}{|c|c|c|c|c|}
\hline $\begin{array}{l}\text { Patient } \\
\text { Number }\end{array}$ & $\begin{array}{l}\text { Calculated Cerebrospinal } \\
\text { Fluid Phenytoin } \\
\text { Concentration ( } \mu \mathrm{g} / \mathrm{mL})\end{array}$ & $\begin{array}{l}\text { Calculated Weight- } \\
\text { Based Phenytoin } \\
\text { Dose (mg) }\end{array}$ & $\begin{array}{l}\text { Difference Between } \\
\text { Calculated and Administered } \\
\text { Phenytoin Dose (mg) }\end{array}$ & $\begin{array}{l}\text { Difference Between Goal Phenytoin } \\
\text { Concentration and Measured } \\
\text { Concentration ( } \mu \mathrm{g} / \mathrm{mL} \text { ) }\end{array}$ \\
\hline P2-1 & 0.0 & 935 & 65 & -2.0 \\
\hline P2-2 & 2.8 & 935 & 65 & 0.8 \\
\hline P2-3 & 2.3 & 935 & 65 & 0.3 \\
\hline P8-1 & 0.0 & 1853 & -853 & -2.0 \\
\hline P8-2 & 1.5 & 1853 & -853 & -0.5 \\
\hline P8-3 & 0.6 & 1853 & -853 & -1.4 \\
\hline P11-1 & 0.0 & 1309 & -309 & -2.0 \\
\hline P11-2 & 0.7 & 1309 & -309 & -1.3 \\
\hline P11-3 & 1.0 & 1309 & -309 & -1.0 \\
\hline P14-1 & 0.0 & 1207 & -207 & -2.0 \\
\hline P14-2 & 3.2 & 1207 & -207 & 1.2 \\
\hline P14-3 & 2.5 & 1207 & -207 & 0.5 \\
\hline P15-1 & 0.0 & 1326 & -326 & -2.0 \\
\hline P15-2 & 3.7 & 1326 & -326 & 1.7 \\
\hline P15-3 & 1.4 & 1326 & -326 & -0.6 \\
\hline P21-1 & 0.0 & 1088 & -88 & -2.0 \\
\hline P21-2 & 1.0 & 1088 & -88 & -1.0 \\
\hline P21-3 & 1.5 & 1088 & -88 & -0.5 \\
\hline
\end{tabular}

\section{TABLE 1: Cerebrospinal fluid phenytoin concentration in $\mu \mathrm{g} / \mathrm{mL}$ measured by high performance} liquid chromatography

Abbreviations: P: patient followed by patient number and sample number. Sample one as obtained prior to administration of antiepileptic, sample two was obtained one hour after administration of antiepileptic, and sample three was obtained six hours after administration of antiepileptic. 


\section{Cureus}

\begin{tabular}{|c|c|c|c|c|}
\hline $\begin{array}{l}\text { Patient } \\
\text { number }\end{array}$ & $\begin{array}{l}\text { Calculated } \\
\text { Levetiracetam } \\
\text { Concentration } \\
(\mu \mathrm{g} / \mathrm{mL})\end{array}$ & $\begin{array}{l}\text { Calculated Weight- } \\
\text { Based Levetiracetam } \\
\text { Dose (mg) }\end{array}$ & $\begin{array}{l}\text { Difference Between Calculated } \\
\text { and Administered } \\
\text { Levetiracetam Dose }(\mathrm{mg})\end{array}$ & $\begin{array}{l}\text { Difference Between Goal } \\
\text { Levetiracetam Concentration and } \\
\text { Measure Concentration }(\mu \mathrm{g} / \mathrm{mL})\end{array}$ \\
\hline P6-1 & 0.0 & 1860 & -860 & -12.0 \\
\hline P6-2 & 4.5 & 1860 & -860 & -7.5 \\
\hline P6-3 & 15.4 & 1860 & -860 & 3.4 \\
\hline P10-1 & 0.1 & 1360 & -360 & -11.9 \\
\hline P10-2 & 17.0 & 1360 & -360 & 5.0 \\
\hline P10-3 & 15.6 & 1360 & -360 & 3.6 \\
\hline P12-1 & 0.1 & 1760 & -760 & -11.9 \\
\hline P12-2 & 7.8 & 1760 & -760 & -4.2 \\
\hline P12-3 & 7.8 & $1 / 60$ & -160 & -4.2 \\
\hline P13-1 & 0.2 & 1900 & -900 & -11.8 \\
\hline P13-2 & 7.9 & 1900 & -900 & -4.1 \\
\hline P13-3 & 7.6 & 1900 & -900 & -4.4 \\
\hline P18-1 & 0.0 & 1180 & -180 & -12.0 \\
\hline P18-2 & 11.2 & 1180 & -180 & -0.8 \\
\hline P18-3 & 6.6 & 1180 & -180 & -5.4 \\
\hline P20-1 & 0.0 & 1560 & -560 & -12.0 \\
\hline P20-2 & 13.3 & 1560 & -560 & 1.3 \\
\hline P20-3 & 10.5 & 1560 & -560 & -1.5 \\
\hline
\end{tabular}

TABLE 2: Difference between measured concentration of levetiracetam in the CSF $(\square \mathrm{g} / \mathrm{mL}$ ) with goal concentration

Abbreviations: P: patient followed by patient number and sample number. Sample one as obtained prior to administration of antiepileptic, sample two was obtained one hour after administration of antiepileptic, and sample three was obtained six hours after administration of antiepileptic.

Differences between actual dose and weight-based dosing of phenytoin and levetiracetam compared to goal six-hour CSF concentrations of levetiracetam and phenytoin are plotted below in Figures 1-2. On the y-axis, positive values are those above minimum threshold CSF concentrations and negative values are those below minimum threshold CSF concentrations for phenytoin and levetiracetam, $2.0 \mu \mathrm{g} / \mathrm{mL}$ and $12.0 \mu \mathrm{g} / \mathrm{mL}$, respectively. Further positive values on the $\mathrm{x}$-axis represent doses of antiepileptic greater than goal dose while those with negative values represent the milligrams less than goal dose the patient received.

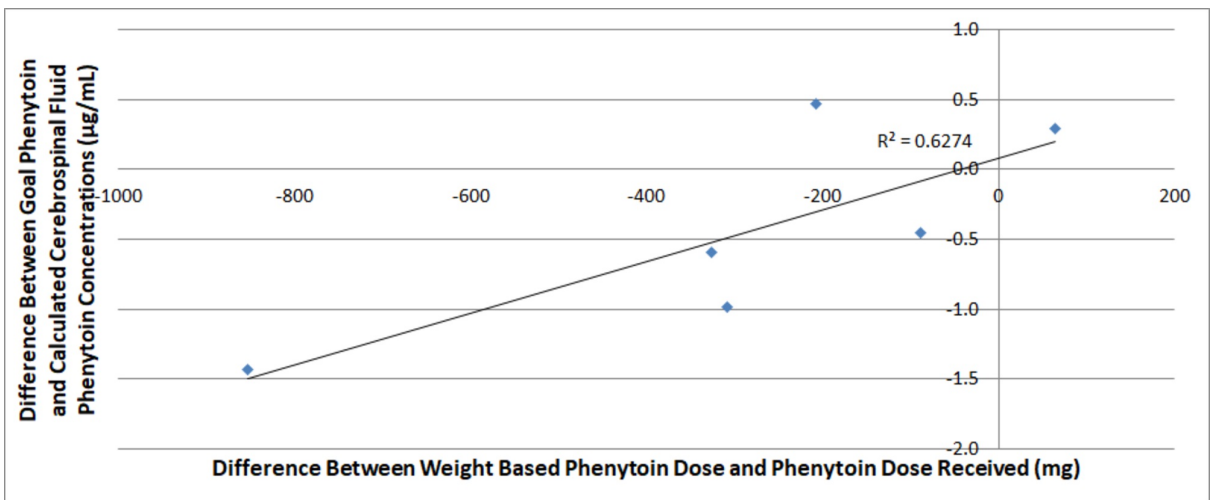


FIGURE 1: A comparison of weight-based phenytoin dosing compared to differences between goal and calculated cerebrospinal fluid phenytoin concentrations six hours after dosage

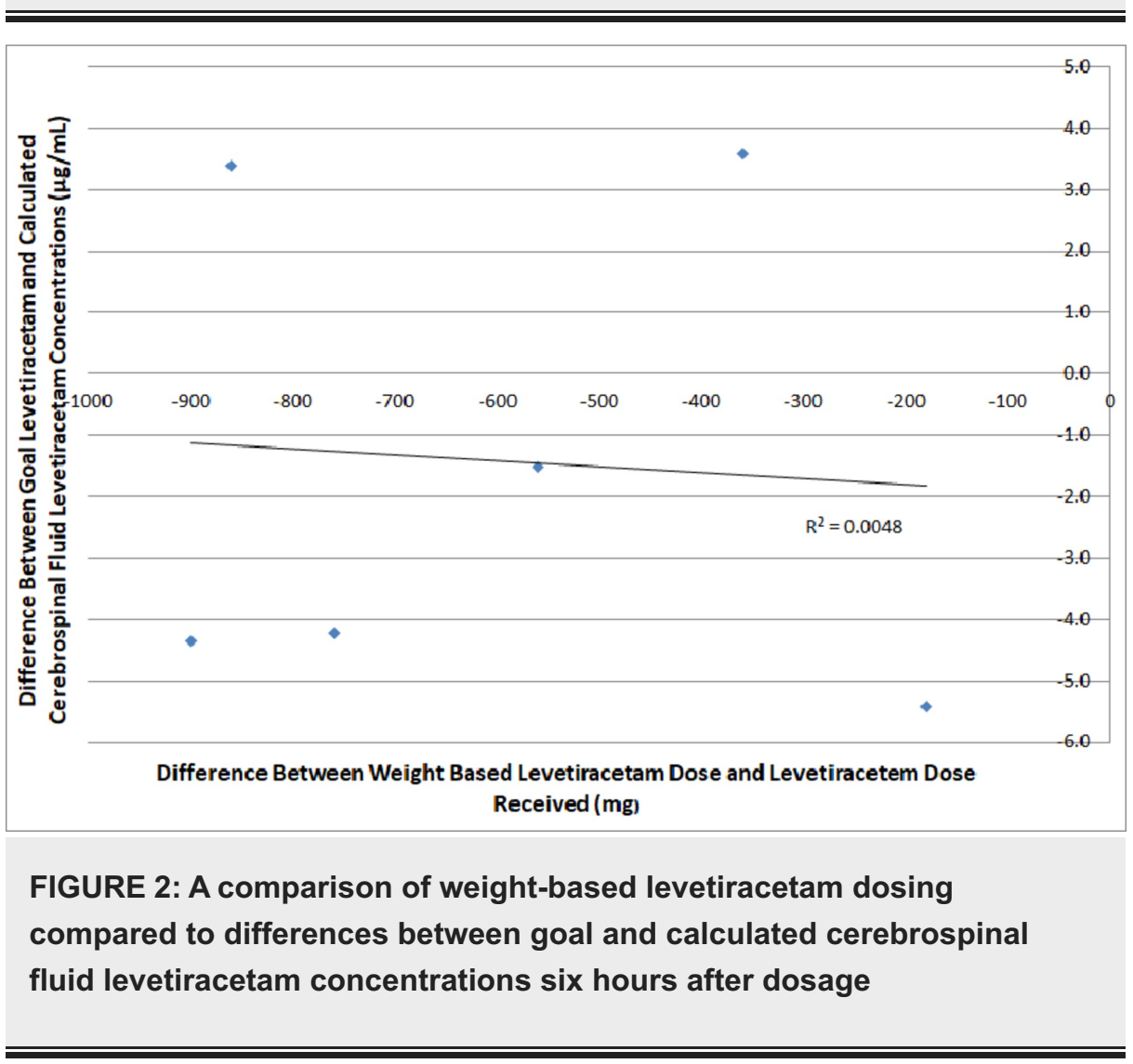

Figures 1-2 identify the difference between the administered and calculated dosing of antiepileptic and goal and calculated CSF concentrations of the antiepileptic. Figure 2 shows that there was not a predictable trend correlating variances or proximity of dosing to goal weight-based dose of levetiracetam to variances in the measured to goal CSF concentration. Figure 1 identifies a trend in which the closer the patient's dose was to the weight-based goal phenytoin, the more their CSF phenytoin level increased with an R-squared value of 0.6274 .

\section{Discussion}

The current literature demonstrates that levetiracetam, when recommended blood level doses are reached, can significantly increase the seizure-free time period following post-traumatic brain injury [15].

Recommended levetiracetam dosage ranges from 1000 - $4000 \mathrm{mg} /$ day, with median dosage beginning at $3000 \mathrm{mg} /$ day [13-15]. The therapeutic range of levetiracetam has not yet been definitively defined, however, a trough level between 12 and $46 \mu \mathrm{g} / \mathrm{mL}$ has been suggested in the literature [16-18].

A variety of laboratory methods have been utilized to measure levetiracetam levels in biological matrices, including but not limited to, immunoassays, HPLC with UV detection, gas chromatography with mass spectrometry detection, high-performance liquid chromatography-tandem mass spectrometry, capillary electrophoresis with UV detection, and ultra-performance liquid chromatography tandem-mass spectrometry [12]. Some of these methods require large sample volumes or lengthy run times to determine levetiracetam concentrations.

Our patient population consisted of two groups of six patients with sTBI who underwent EVD placement. For seizure prophylaxis, these two groups received levetiracetam and phenytoin, respectively. Studies have shown that seizures within the first-week post-traumatic brain injury can be decreased with AED administration [19]. Further studies have also explored the differences between levetiracetam and phenytoin as AEDs of choice [20]. Our study sought to evaluate the differences in CSF concentration between levetiracetam and phenytoin. We further sought to evaluate if standard dosage regimens were appropriate as compared to a weight-based dosage regime. With the placement of EVDs, we were able to draw CSF in order to evaluate the levels of AEDs present within the CSF. This was done via HPLC. Goal concentrations were 
selected as $2 \mu \mathrm{g} / \mathrm{mL}$ for phenytoin and $12 \mu \mathrm{g} / \mathrm{mL}$ for levetiracetam [12-13]. Calculated levels of phenytoin and levetiracetam were made, correcting from baseline measurements of CSF prior to AED administration. Also demonstrated were the difference between weight-based calculations and our standard dosing regimens used for seizure prophylaxis (Tables $1-2$ ).

The current approach to antiepileptic prophylaxis involves the administration of either intravenous or oral medications to produce high levels of the medications in the bloodstream. Regardless of the administration, the medication must travel from the bloodstream to the brain tissue. The largest obstacle with the administration of such medications is the blood-brain barrier (BBB), which is composed of tight junctions between endothelial cells, with an absence of fenestrations and strict regulation of molecules from the blood into the brain interstitial fluid. Given this challenge, there are several new approaches to increase the entry and persistence of antiepileptic medications in the brain, including strategies such as drug delivery systems, prodrugs, efflux pump inhibition, hyperosmolar BBB opening, and circumventing the BBB via direct drug delivery to the ventricle and cortex [10].

Our study suggests that phenytoin reaches appropriate CSF concentration levels more predictably than those of levetiracetam when doses approximating weight-based calculations are used. As phenytoin levels approached weight-based dosing, CSF levels increased toward the therapeutic range. This relationship was not appreciated when plotting CSF levetiracetam levels against given and weight-based dosages (Figures 1-2). Although both groups did have loading doses less than the calculated weight-based doses, only the phenytoin group demonstrated predictability in measured CSF concentrations. Altogether, this suggests that appropriate CSF concentrations may be more easily achieved using phenytoin over levetiracetam intravenously.

Some of the limitations associated with this study have been identified. Data were collected from only one level II trauma center over a year-long period. We appreciate that, with just one center participating in the study, center-specific patient characteristics may contribute to the outcome. Furthermore, this study evaluated the CSF concentrations and pharmacodynamics of selected AED therapy but did not investigate the outcomes or incidence of post-traumatic seizure in this population. Further studies could be conducted to investigate this. Additionally, our study would benefit from larger sample sizes.

\section{Conclusions}

The administration of AEDs are paramount to the prevention of seizure following TBI. Our study suggests that phenytoin reaches appropriate CSF concentration levels over that of levetiracetam when doses approximating weight-based calculations are used. Also, a correlation was found between the weight-based dosing of AED and higher concentration in CSF. More studies will be required in the future to determine the significance of this relationship.

\section{Additional Information \\ Disclosures}

Human subjects: Consent was obtained by all participants in this study. Teckah Lawrence, M.Ed issued approval NA. This study was approved by the ARMC IRB Committee. Animal subjects: All authors have confirmed that this study did not involve animal subjects or tissue. Conflicts of interest: In compliance with the ICMJE uniform disclosure form, all authors declare the following: Payment/services info: All authors have declared that no financial support was received from any organization for the submitted work. Financial relationships: All authors have declared that they have no financial relationships at present or within the previous three years with any organizations that might have an interest in the submitted work. Other relationships: All authors have declared that there are no other relationships or activities that could appear to have influenced the submitted work.

\section{References}

1. Inaba K, Menaker J, Branco BC, et al.: A prospective multicenter comparison of levetiracetam versus phenytoin for early posttraumatic seizure prophylaxis. J Trauma Acute Care Surg. 2013, 74:766-773. 10.1097/TA.0b013e3182826e84

2. Rutland-Brown W, Langlois JA, Thomas KE, Xi YL: Incidence of traumatic brain injury in the United States . J Head Trauma Rehabil. 2006, 21:544-548.

3. Temkin NR, Dikmen SS, Wilensky AJ, et al.: A randomized, double-blind study of phenytoin for the prevention of post-traumatic seizures. New Eng J Med. 1990, 323:497-502. 10.1056/NEJM199008233230801

4. Brain Trauma Foundation, American Association of Neurological Surgeons, Congress of Neurological Surgeons: Guidelines for the management of severe traumatic brain injury 3rd edition . J Neurotrauma. 2007, 24:i-vi. 10.1089/neu.2007.9999

5. Kruer RM, Harris LH, Goodwin H, Kornbluth J, Thomas KP, Slater LA, Haut ER: Changing trends in the use of seizure prophylaxis after traumatic brain injury: a shift from phenytoin to levetiracetam. J Crit Care. 2013, 28:883-889. 10.1016/j.jcrc.2012.11.020

6. Patanwala AE, Kurita A, Truong E: Low-dose levetiracetam for seizure prophylaxis after traumatic brain injury. Brain Inj. 2016, 30:156-158. 10.3109/02699052.2015.1089596

7. Walia KS, Khan EA, Ko DH, Raza SR, Khan YN: Side effects of antiepileptics-a review . Pain Practice. 2004, 
4:194-203. 10.1111/j.1533-2500.2004.04304.x

8. Harris L, Hateley S, Seemungal B: P22 phenytoin versus levetiracetam for post traumatic brain injury seizure prophylaxis; a retrospective study at a UK major trauma centre. J Neurol Neurosurg Psychiatry. 2019, 90:e31. 10.1136/jnnp-2019-ABN.98

9. Bhullar IS, Johnson D, Paul JP, Kerwin A, Tepas J, Frykberg E: More harm than good: anti-seizure prophylaxis after traumatic brain injury does not decrease seizure rates but may inhibit functional recovery. J Trauma Acute Care Surg. 2014, 76:54-61. 10.1097/TA.0b013e3182aafd15

10. Ramael S, Daoust A, Otoul C, Toublanc N, Troenaru M, Lu Z, Stockis A: Levetiracetam intravenous infusion: a randomized, placebo-controlled safety and pharmacokinetic study. Epilepsia. 2006, 47:1128-1135. 10.1111/j.1528-1167.2006.00586.x

11. Bennewitz MF, Saltzman WM: Nanotechnology for delivery of drugs to the brain for epilepsy . Neurotherapeutics. 2009, 6:323-336. 10.1016/j.nurt.2009.01.018

12. Koren G, Barzilay Z, Schachar E, Brand N, Danee S, Halkin H, MacLeod SM: Kinetics of CSF phenytoin in children. Can J Neurol Sci. 1983, 10:195-197.

13. McDonald D, Sultan R, Viswanathan A, Siu A: A rare case of levetiracetam and drug-induced idiopathic aseptic meningitis in a pediatric patient. Pharmacotherapy. 2019, 39:109-113. 10.1002/phar.2198

14. Trinka E, Dobesberger J: New treatment options in status epilepticus . Ther Adv Neurol Disord. 2009, 2:7991. $10.1177 / 1756285608100460$

15. Stepanova D, Berang RG: Measurements of levetiracetam drug levels to assist with seizure control and monitoring of drug interactions with other anti-epileptic medications (AEMs). Seizure. 2014, 23:371-376. 10.1016/j.seizure.2014.02.003

16. Abou-Khalil B: Levetiracetam in the treatment of epilepsy. Neuropsychiatr Dis Treat. 2008, 4:507-523. 10.2147/ndt.s2937

17. Hirsch LJ, Arif H, Buchsbaum R, et al.: Effect of age and co-medication on levetiracetam pharmacokinetics and tolerability. Epilepsia. 2007, 48:1351-1359. 10.1111/j.1528-1167.2007.01043.x

18. De Smedt T, Raedt R, Vonck K, Boon P: Levetiracetam. Part II. The clinical profile of a novel anticonvulsant drug. CNS Drug Rev. 2007, 13:57-78. 10.1111/j.1527-3458.2007.00005.x

19. Carney N, Totten AM, O'Reilly C, et al.: Guidelines for the management of severe traumatic brain injury, fourth edition. Neurosurgery. 2017, 80:6-15. 10.1227/NEU.0000000000001432

20. Szaflarski JP, Sangha KS, Lindsell CJ, Shutter LA: Prospective, randomized, single-blinded comparative trial of intravenous levetiracetam versus phenytoin for seizure prophylaxis. Neurocrit Care. 2010, 12:165-172. 